in vivo $34: 3681-3685(2020)$

doi:10.21873/invivo.12216

\title{
Transapical Left Ventricular Approach for Cardiac Papillary Fibroelastomas: A Case Report
}

\author{
OVIDIU STIRU ${ }^{1,2^{*}}$, ROXANA CARMEN GEANA ${ }^{1 *}$, PETRU RAZVAN DRAGULESCU $^{1 *}$, ADRIAN TULIN $^{3,4^{*}}$, \\ LAURA RADUCU $^{5,6^{*}}$, NICOLAE BACALBASA ${ }^{7,8,9}$, IRINA BALESCU ${ }^{10}$, DRAGOS CRETOIU ${ }^{11,12}$, \\ CAMELIA DIACONU ${ }^{13,14}$, LAURA ILIESCU ${ }^{13,15}$, CORNEL SAVU ${ }^{2,16}$ and VLAD ANTON ILIESCU ${ }^{1,2}$ \\ ${ }^{1}$ Prof. Dr. C. C. Iliescu Emergency Institute for Cardiovascular Diseases, Bucharest, Romania; \\ ${ }^{2}$ Department of Cardio-Thoracic Pathology, Carol Davila University of Medicine and Pharmacy, Bucharest, Romania; \\ ${ }^{3}$ Department of Anatomy, Carol Davila University of Medicine and Pharmacy, Bucharest, Romania; \\ ${ }^{4}$ Department of General Surgery, Prof. Dr. Agripa Ionescu, Clinical Emergency Hospital, Bucharest, Romania; \\ ${ }^{5}$ Department of Plastic and Reconstructive Microsurgery, \\ Carol Davila University of Medicine and Pharmacy, Bucharest, Romania; \\ ${ }^{6}$ Department of Plastic and Reconstructive Surgery, Prof Dr. Agripa Ionescu, \\ Clinical Emergency Hospital, Bucharest, Romania; \\ ${ }^{7}$ Department of Obstetrics and Gynecology, \\ Carol Davila University of Medicine and Pharmacy, Bucharest, Romania; \\ ${ }^{8}$ Department of Obstetrics and Gynecology, I. Cantacuzino Clinical Hospital, Bucharest, Romania; \\ ${ }^{9}$ Department of Visceral Surgery, Center of Excellence in Translational Medicine, \\ Fundeni Clinical Institute, Bucharest, Romania; \\ ${ }^{10}$ Department of Surgery, Ponderas Academic Hospital, Bucharest, Romania; \\ ${ }^{11}$ Department of Cellular, Molecular and Histology Biology, \\ Carol Davila University of Medicine and Pharmacy, Bucharest, Romania; \\ ${ }^{12}$ Scientific researcher, Alessandrescu-Rusescu National Institute of Mother and Child Health, \\ Fetal Medicine Excellence Research Center, Bucharest, Romania; \\ ${ }^{13}$ Department of Internal Medicine, Carol Davila University of Medicine and Pharmacy, Bucharest, Romania; \\ ${ }^{14}$ Department of Internal Medicine, Clinical Emergency Hospital of Bucharest, Bucharest, Romania; \\ ${ }^{15}$ Department of Internal Medicine, Fundeni Clinical Institute, Bucharest, Romania; \\ ${ }^{16}$ Department of Thoracic Surgery, Marius Nasta Institute of Pneumonology, Bucharest, Romania
}

\begin{abstract}
Background/Aim: Cardiac papillary fibroelastomas $(C P F)$ are benign tumors, frequently asymptomatic, characterized by a mobile pedunculated mass that arises from the endocardium. When CPF is located in the left ventricle, it may protrude into the left ventricular outflow tract and affect hemodynamics. They are highly thrombogenic, and can also cause some life-threatening events such as cerebral and
\end{abstract}

This article is freely accessible online.

*These Authors contributed equally to this study.

Correspondence to: Nicolae Bacalbasa, Carol Davila University of Medicine and Pharmacy, Bucharest, Romania. Tel: +40 723540426, e-mail: nicolae_bacalbasa@yahoo.ro

Key Words: Cardiac papillary fibroelastomas, left ventricle, surgery. peripheral embolization. Case Report: We herein report a case of a 74-year-old female admitted to our center with palpitations and dyspnea on exertion. Her past medical history revealed that she had had a transient ischemic attack 7 months before presentation. Echocardiography and cardiac magnetic resonance imaging revealed an intracardiac mass anchored in the anteroapical interventricular septum without interference with aortic or mitral valve functionality. Surgical resection of the left ventricular mass was performed through the left apical ventriculotomy approach. Histopathological examination of the tumor was suggestive of papillary fibroelastoma. The postoperative course was uneventful. The patient was discharged home on the eighth postoperative day, with no recurrence at 6 months. Conclusion: Although left ventricular papillary fibroelastomas are benign tumors, they carry a high risk for embolic complications and therefore surgery should be proposed, the transapical approach being a safe and effective method. 

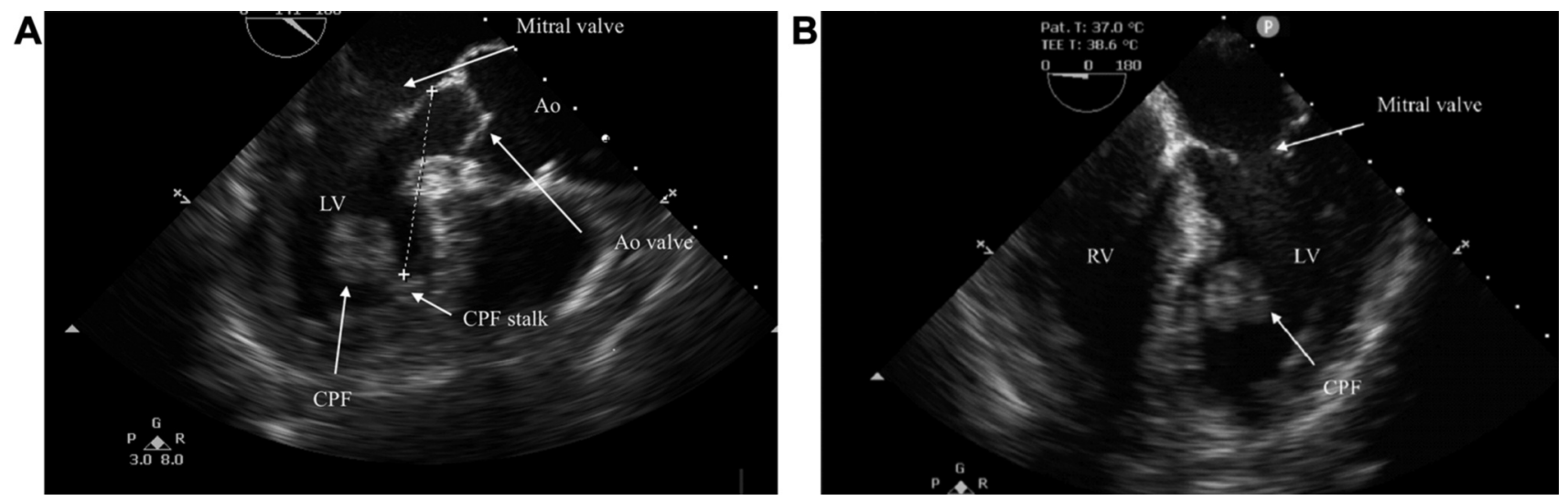

Figure 1. A: Transthoracic echocardiography (apical four-chamber) showing a mobile, tumoral mass (arrow) in the left ventricle (LV) and a stalk attached to the anteroapical interventricular septum. B: Transthoracic echocardiography (long-axis view) showing no interference with mitral and aortic valves. Ao: Aorta; CPF : cardiac papillary fibroelastoma; LV: left ventricle; RV: right ventricle.

Cardiac papillary fibroelastoma (CPF) is the second most common primary cardiac tumor in adults (1), the most common being myxomas (2), and followed by lipomas (3). $\mathrm{CPF}$ has an incidence of $0.02 \%$ in every one million autopsies (4). They are usually discovered as solitary tumors; multiple tumors are relatively rare (9\%) (4). CPFs are small and friable slow-growing tumors with multiple avascular papillary fronds made of collagen, elastic fibers, and proteoglycans. Their pathogenesis remains uncertain (5). Although histologically benign, papillary fibroelastomas can lead to life-threatening complications from the embolization of fragments in systemic and pulmonary circulation depending on their dimension, mobility, and location. In more than $80 \%$ of patients, CPFs are located on left-sided cardiac valves (4). CPF incidence was found to be higher in males compared to females; the percentage of asymptomatic patients was $30 \%$ (reviewed in (4)]. Although histologically benign, $\mathrm{CPF}$ has lethal potential due to impairment of cardiac dynamics and embolization of fragments into the coronary arteries, systemic circulation, and pulmonary circulation (6). The described report illustrates our surgical approach in a case of left ventricular papillary fibroelastoma.

\section{Case Report}

After obtaining the approval of the Ethical Committee (no. 79/June 2020), we report the case of a 74-year-old woman presenting at our center for the treatment of a cardiac mass discovered during investigation for palpitations, dyspnea on exertion, dizziness, and fatigue, which had progressively worsened over the past month. The onset of symptoms was 1 year earlier. The patient was hypertensive and dyslipidemic. The patient had a history of transient ischemic attack 7 months earlier. She had taken antihypertensives and lipid-lowering

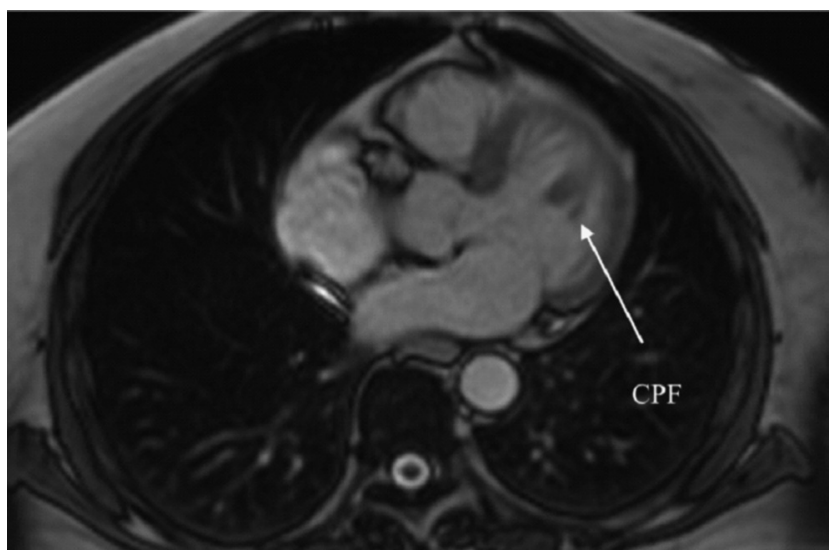

Figure 2. Cardiac magnetic resonance imaging of the heart revealed a $1.5 \mathrm{~cm}$ pedunculated mass with intermediate signal intensity on T1weighted images.

medications. On admission to our cardiac center, the patient's blood pressure, heart rate and temperature were normal. A chest $\mathrm{X}$-ray at admission showed a normal cardiac silhouette, and the electrocardiogram showed normal sinus rhythm. Laboratory findings, including complete hematological and biochemical tests, were all within normal limits with no increase in the inflammatory index (C-reactive protein, white blood cell count). Transthoracic and transesophageal echocardiography revealed an echo-dense, mobile mass in the left ventricle of $1.5 \times 1.6 \mathrm{~cm}$ in diameter, originating from the anteroapical wall of the interventricular septum, without protruding into the mitral valve or left ventricular outflow tract (Figure 1). Left ventricular ejection fraction was normal, without any valvular pathology. Color Doppler ultrasound of the carotid artery showed nondiseased arteries. Coronary angiography demonstrated normal coronary arteries. Following the transthoracic echocardiogram 


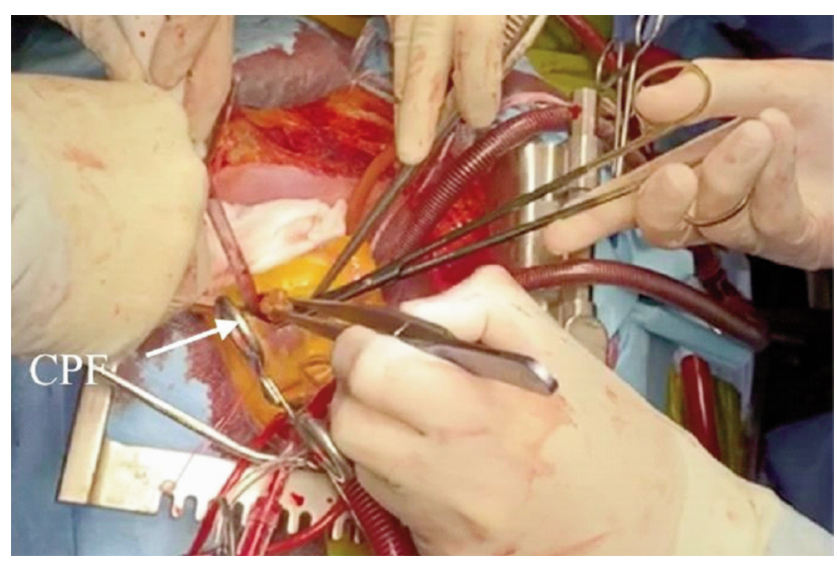

Figure 3. Surgical view with an approach through the left apical ventricle, with cardiac papillary fibroelastoma adhered to the interventricular septum.

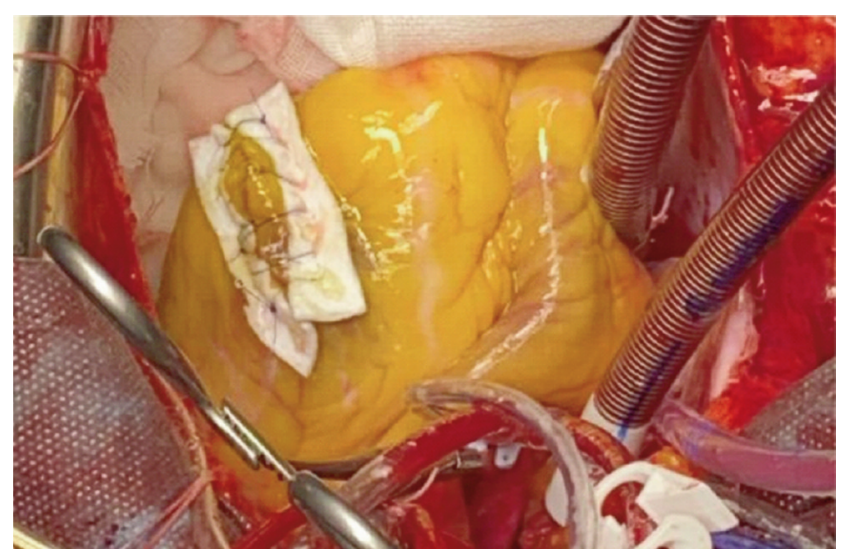

Figure 4. Surgical view. The ventriculotomy was closed by double-layer running Prolene 4/0 stitches with underlying Teflon felt strips.

(TTE) examination, cardiac magnetic resonance imaging (CMR) was then performed to determine the character of the tumor tissue. CMR confirmed the presence in the left ventricular cavity of a $2-\mathrm{cm}$ mobile pedunculated mass with intermediate signal intensity on T1-weighted images (Figure 2).

Both TTE and CMR revealed that the tumor was mobile, located in the left ventricle, and had a 5-7 mm narrow stalk, originating from the anteroapical wall of the interventricular septum. The management of the case was led by the patient's history of systemic embolization. The risks of the surgery and expected natural history without surgery were communicated to the patient. The patient agreed to surgical treatment. The surgical strategy was discussed considering the transesophageal echocardiography (TEE), TTE, and CMR imagery, and planned immediately because of the hypermobility of the mass that potentially was able to cause a

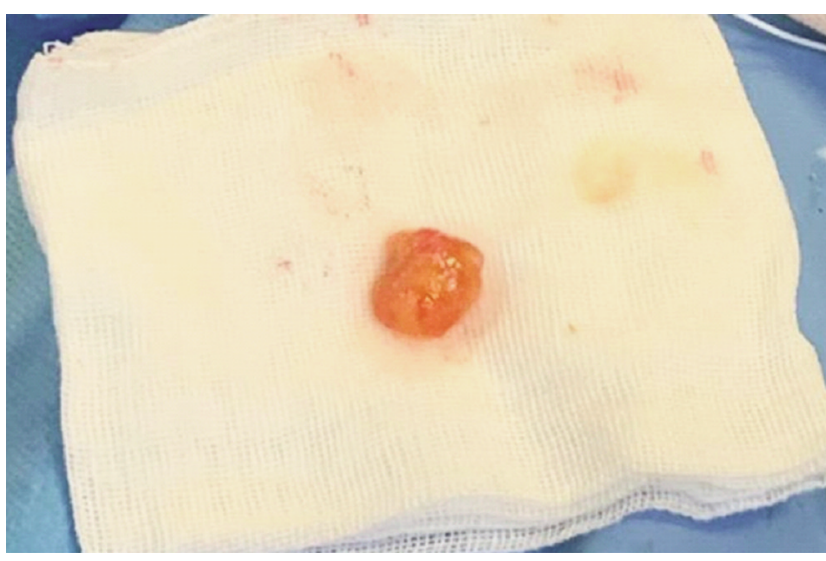

Figure 5. On gross pathological examination, the cardiac papillary fibroelastoma was an ovoid, soft, gelatinous, white fragment of tissue $(1.4 \times 1.5 \mathrm{~cm})$.

fatal cerebral infarction. The surgery was performed through a median sternotomy; cardiopulmonary bypass was established by cannulating the ascending aorta and right atrium with a venting tube placed in the left atrium through the right superior pulmonary vein. After aortic cross-clamping, cardioplegia was immediate by delivery of a single injection of cold $\left(4^{\circ} \mathrm{C}\right)$ blood cardioplegic solution into the aortic root. A $3 \mathrm{~cm}$ left apical ventriculotomy was made parallel to the posterior descending coronary artery at $2 \mathrm{~cm}$ strictly to the boundary. This approach allowed us to visualize the tumor easily. The intraoperative finding revealed a pedunculated mobile tumoral mass attached to the antroapical interventricular septum, which was excised (Figure 3). The ventriculotomy was closed by double-layer running Prolene 4/0 stitches with underlying Teflon felt strips (Figure 4). Total cardiopulmonary bypass time was 32 minutes, and the aorta cross-clamp time was 24 minutes. The patient was successfully weaned from cardiopulmonary bypass with a small dose of vasoconstrictor drugs. The tumor was sent for pathological examination. Postoperative echocardiography showed normal coaptation of the mitral and aortic valve without leaflet injury and with no left ventricular fraction impairment.

At the gross pathological examination, the tumor was found to be an ovoid, soft, gelatinous, white fragment of tissue (Figure 5). The histopathological findings showed multiple elongated and branching papillary fronds, consisting of an acellular matrix surrounded by a single layer of endothelial cells, and led to the diagnosis of CPF (Figure 6).

The postoperative course was uneventful. The patient was discharged home on the eighth postoperative day, with an improvement of her clinical state and effort tolerance. At the 6-month follow-up, TTE was normal, and no recurrence was observed. 


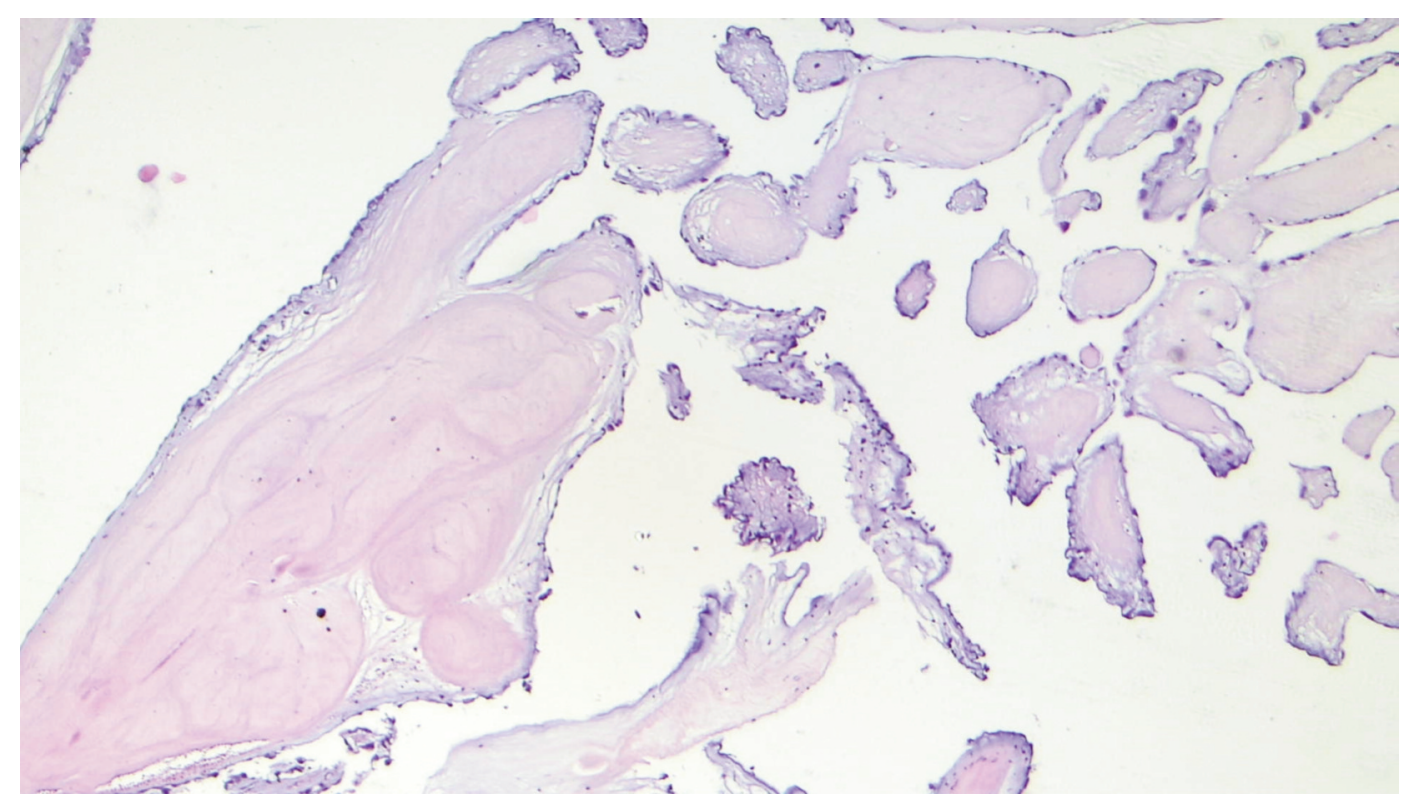

Figure 6. The histopathological findings showing multiple elongated and branching papillary fronds, consisting of an acellular matrix surrounded by a single layer of endothelial cells, led to the diagnosis of cardiac papillary fibroelastoma (hematoxylin and eosin staining, $\times 10$ ).

\section{Discussion}

$\mathrm{CPF}$ is a gelatinous tumor derived from the endocardial surfaces, including papillary muscles, tendinous chords, the septum, or free walls of any of the cardiac chambers (6). The age of patients is variable, most cases of CPF are described in adult patients over the age of 50 years, and there is no difference between both sexes (7). Gowda et al. reported that in $90 \%$ of cases cardiac valves were affected, with $44 \%$ being found in aortic valves, followed by $35 \%$ in the mitral valves (4). CPFs rarely occur as single lesions and are smaller than $1 \mathrm{~cm}$ in diameter (7). Clinically, most CPF may be fully asymptomatic and often discovered incidentally in cardiac imaging. In most cases, the slow growth of the tumor often delays diagnosis.

The clinical presentation described features typical for cardioembolism, neurological or cardiac complications, including transient ischemic attack, stroke, angina, myocardial infarction, sudden death, heart failure, syncope, and blindness $(8,9)$. The differential diagnosis should consider vegetation associated with endocarditis, myxoma, or histiocytoma (10). In cases of CPF, Tsugu et al. reported a higher incidence of embolism than with other benign cardiac tumors such as myxoma, lipoma, or even vegetative lesions (11). The diagnosis is usually accomplished through TEE and CMR. Single CPFs are detected by TTE in $91.4 \%$ of cases, whereas multiple fibroelastomas are only detected in $8.6 \%$ (12). Small CPFs are difficult to see on TEE, CMR providing better visualization of cardiac tumors when TEE is inconclusive. However, both are currently used as a method of diagnosing cardiac tumors (13). In our case, CMR led to defining the tumor presence, origin and excluded the presence of a second tumor. Although there are no guidelines on the management of CPF, there is a general agreement on treating patients with surgery. Surgery is indicated for all symptomatic fibroelastomas. However, in asymptomatic patients, Gowda et al. recommends conservative treatment without the need for permanent anticoagulation (4). In our case, the patient had a freely mobile left ventricular mobile papillary fibroelastoma with history of embolization, and we considered surgical treatment as an emergency. Depending on tumor size and location, the surgical approach is the key to intervention (14-18). However, some articles in the literature recommend ascending aortic or transvalvular mitral approach for $\mathrm{CPF}$ resection $(6,19)$. In our case, due to the apical ventricular localization, friability and relatively large tumor size, we chose the left ventricular apical approach. It was easy to perform, does not require special technical skills, and has no impairment of left ventricular function on TEE examination. In the literature, there is also the opinion that asymptomatic patients with small left-sided nonmobile lesions must be closely followed up with echocardiography until they enlarge and become mobile, at which time they can then be referred to surgery (20). Despite the lack of guidelines, we agree that fragile patients who are not candidates for surgical resection should be treated with long-term anticoagulation (20). 


\section{Conclusion}

Although left ventricular papillary fibroelastomas are benign, they carry a high risk for embolic complications. Once CPF is diagnosed in the left ventricle without interfering with the ventricle's ejection tract or mitral valve functionality, we recommend early surgical referral. The transapical left ventricular approach was technically easy to perform through a small incision, did not require special technical skills, and did not impair left ventricular function.

\section{Conflicts of Interest}

There are no conflicts of interest to declare regarding this study.

\section{Authors' Contributions}

OS, RCG, PRD, VAI performed the surgical procedure; AT, LR, $\mathrm{NB}$, DC, CS reviewed literature data; CD, LI preoperative investigation the patient, IB, NB, OS prepared the draft of the article; VAI was advisor of the surgical procedures OS, NB, VAI reviewed the final version of the article. All Authors read and approved the final version of the article.

\section{References}

1 Edwards FH, Hale D, Cohen A, Thompson L, Pezzella AT and Virmani R: Primary cardiac valve tumors. Ann Thorac Surg 52(5): 1127-1131, 1991. PMID: 1953134. DOI: 10.1016/00034975(91)91293-5

2 Iliescu VA, Dorobantu LF, Stiru O, Iosifescu AG, Coman I, Marin S and Filipescu D: Second recurrence of cardiac myxoma 7 years after the initial operation. Chirurgia 103(2): 239-241, 2008. PMID: 18457107.

3 McAllister HA, Fenoglio JJ: Tumors of the Cardiovascular System. In: Atlas of Tumor Pathology. Armed Forces Institute of Pathology (Second Series), Washington, D.C., 1978.

4 Gowda RM, Khan IA, Nair CK, Mehta NJ, Vasavada BC and Sacchi TJ: Cardiac papillary fibroelastoma: A comprehensive analysis of 725 cases. Am Heart J 146(3): 404-410, 2003. PMID: 12947356. DOI: 10.1016/S0002-8703(03)00249-7

5 Parthenakis F, Nyktari E, Patrianakos A, Pitsis A, Asimaki A and Vardas P: Asymptomatic papillary fibroelastoma of the aortic valve in a young woman - a case report. Cardiovasc Ultrasound 7: 43, 2009. PMID: 19725951. DOI: 10.1186/1476-7120-7-43

6 Tanaka H, Narisawa T, Mori T, Masuda Y and Kishi D: Double primary left ventricular and aortic valve papillary fibroelastoma. Circ J 68(5): 504-506, 2004. PMID: 15118297. DOI: 10.1253/ circj.68.504

7 Pacini D, Farneti PA, Leone O and Galli R: Cardiac papillary fibroelastoma of the mitral valve chordae. Eur J Cardiothorac Surg 13(3): 322-324, 1998. PMID: 9628387. DOI: 10.1016/ s1010-7940(98)00006-2

8 Ngaage DL, Mullany CJ, Daly RC, Dearani JA, Edwards WD, Tazelaar HD, McGregor CG, Orszulak TA, Puga FJ, Schaff HV, Sundt TM, III and Zehr KJ: Surgical treatment of cardiac papillary fibroelastoma: a single center experience with eighty- eight patients. Ann Thorac Surg 80(5): 1712-1718, 2005. PMID: 16242444. DOI: 10.1016/j.athoracsur.2005.04.030

9 Zamora RL, Adelberg DA, Berger AS, Huettner P and Kaplan HJ: Branch retinal artery occlusion caused by a mitral valve papillary fibroelastoma. Am J Ophthalmol 119(3): 325-329, 1995. PMID: 7872394. DOI: 10.1016/s0002-9394(14)71175-4

10 Dorobantu LF, Stiru O, Prodea A, Cioranu R, Georgescu A, Filipescu D and Iliescu VA: Unique case of primary malignant fibrous histiocytoma of the right ventricle with moderator band involvement. Heart Surg Forum 14(4): E245-E248, 2011. PMID: 21859644. DOI: 10.1532/HSF98.20101154

11 Tsugu T, Nagatomo Y, Endo J, Kawakami T, Murata M, Yamazaki M, Shimizu H, Fukuda K, Mitamura H and Lancellotti P: Multiple papillary fibroelastomas attached to left ventricular side and aortic side of the aortic valve: A report of new case and literature review. Echocardiography 36(6): 1194-1199, 2019. PMID: 31116464. DOI: 10.1111/echo.14350

12 Vizzardi E, Faggiano P, Antonioli E, Zanini G, Chiari E, Nodari $\mathrm{S}$ and Cas LD: Thrombus or tumor? A case of fibroelastoma as indicated during the submission process. Cases J 2(1): 31, 2009. PMID: 19133150. DOI: 10.1186/1757-1626-2-31PMID

13 Araoz PA, Mulvagh SL, Tazelaar HD, Julsrud PR and Breen JF: CT and MR imaging of benign primary cardiac neoplasms with echocardiographic correlation. Radiographics 20(5): 1303-1319, 2000. PMID: 10992020. DOI: 10.1148/radiographics.20.5. g00se121303

14 Sousa-Uva M and Cardim N: Cardiac papillary fibroelastoma: So small and yet so dangerous. Rev Port Cardiol 37(12): 987989, 2018. PMID: 30545746. DOI: 10.1016/j.repc.2018.11.006

15 Shi J, Bai ZX, Zhang BG and Guo YQ: Papillary fibroelastoma of the aortic valve in association with rheumatic heart disease: A case report. J Cardiothorac Surg 11: 6, 2016. PMID: 26772603. DOI: 10.1186/s13019-016-0410-6

16 Mkalaluh S, Szczechowicz M, Torabi S, Dib B, Sabashnikov A, Mashhour A, Karck M and Weymann A: Surgery for cardiac papillary fibroelastoma: A 12-year single-institution experience. Med Sci Monit Basic Res 23: 258-263, 2017. PMID: 28706178. DOI: $10.12659 / \mathrm{msmbr} .904881$

17 Abu Saleh WK, Al Jabbari O, Ramlawi B and Reardon MJ: Cardiac papillary fibroelastoma: single-institution experience with 14 surgical patients. Tex Heart Inst J 43(2): 148-151, 2016. PMID: 27127431. DOI: 10.14503/THIJ-14-4889

18 Diaz-Anton B, Gonzalez PA, Parra Jimenez FJ, Cuerpo CG, Perez RF and Solis MJ: Recurrent cardiac fibroelastoma. Is it really a benign tumor? Rev Esp Cardiol 71(8): 685-687, 2018. PMID: 28709859. DOI: 10.1016/j.rec.2017.04.031

19 Akagi H, Irie H, Nakao Y and Sakai K: Transmitral resection of a left ventricular apical papillary fibroelastoma using videoassisted thoracoscopy. J Card Surg 28(6): 651-653, 2013. PMID: 23930599. DOI: 10.1111 jocs. 12190

20 Koniari I, Apostolakis E and Baikoussis NG: eComment: Cardiac papillary fibroelastoma: A current assessment. Interact Cardiovasc Thorac Surg 9(5): 922-923, 2009. PMID: 19828659. DOI: $10.1510 /$ icvts.2009.212274A

Received August 16, 2020

Revised September 7, 2020 Accepted September 10, 2020 